\title{
The multi-messenger approach to particle acceleration by massive stars: a science case for optical, radio and $\mathrm{X}$-ray observatories
}

\author{
Michaël De Becker* \\ Space sciences, Technologies, and Astrophysics Research (STAR) institute, University of Liège, \\ 19c allée du 6 Août, Quartier Agora, B-4000 Liège (Sart-Tilman)
}

\begin{abstract}
Massive stars are extreme stellar objects whose properties allow for the study of some interesting physical processes, including particle acceleration up to relativistic velocities. In particular, the collisions of massive star winds in binary systems lead notably to acceleration of electrons involved in synchrotron emission, hence their identification as non-thermal radio emitters. This has been demonstrated for about 40 objects so far. The relativistic electrons are also expected to produce non-thermal high-energy radiation through inverse Compton scattering. This class of objects permits thus to investigate non-thermal physics through observations in the radio and high energy spectral domains. However, the binary nature of these sources introduces some stringent requirements to adequately interpret their behavior and model non-thermal processes. In particular, these objects are well-established variable stellar sources on the orbital time-scale. The stellar and orbital parameters need to be determined, and this is notably achieved through studies in the optical domain. The combination of observations in the visible domain (including e.g. 3.6-m DOT) with radio measurements using notably GMRT and X-ray observations constitutes thus a promising strategy to investigate particle-accelerating colliding-wind binaries in the forthcoming decade.
\end{abstract}

\section{A few facts}

The massive stars category includes objects with masses higher than about 8-10 solar masses. This category includes O-type, and early B-type stars, along with their evolved counterparts, i.e. WolfRayet (WR) stars and transition objects such as Luminous Blue Variables. A common property of these stars is their powerful and dense stellar winds. Under the strong radiative pressure driven by their high luminosity, the outer layers of these stars are gradually expelled from the stellar surface. This out-flowing material is accelerated to terminal velocities that can be of the order of several $100 \mathrm{~km} \mathrm{~s}^{-1}$ up to a few $1000 \mathrm{~km} \mathrm{~s}^{-1}$. The ejection rate of material through these stellar winds is quite high, with mass loss rates values ranging typically between $10^{-7}$ to $10^{-5} \mathrm{M}_{\odot} \mathrm{yr}^{-1}$, depending on the spectral type and evolutionary stage. Useful references for typical stellar wind parameters are papers published by Muijres et al. (2012) for O-type stars, or by Crowther (2008) for Wolf-Rayet stars.

\footnotetext{
*e-mail: debecker@astro.ulg.ac.be
} 
Among massive stars, a significant fraction are found in binary, or higher multiplicity systems. The binary fraction is not well determined, but a very conservative lower limit is certainly $50 \%$, with an actual fraction very probably significantly higher (Sana \& Evans 2011, Sana et al.2012). In such configurations, the stellar winds will collide - hence the name Colliding-Wind Binary (CWB) - as illustrated in Fig. 1. Among the category of CWBs, a subset is known to be able to accelerate particles up to relativistic velocities. In most cases, the existence of a relativistic electrons population is revealed by synchrotron radio emission. To date, about 40 massive star systems belong to this subset of CWBs, the so-called Particle-Accelerating Colliding-Wind Binaries (PACWBS). The first release of the catalogue of PACWBs has been published by De Becker \& Faucq (2013).

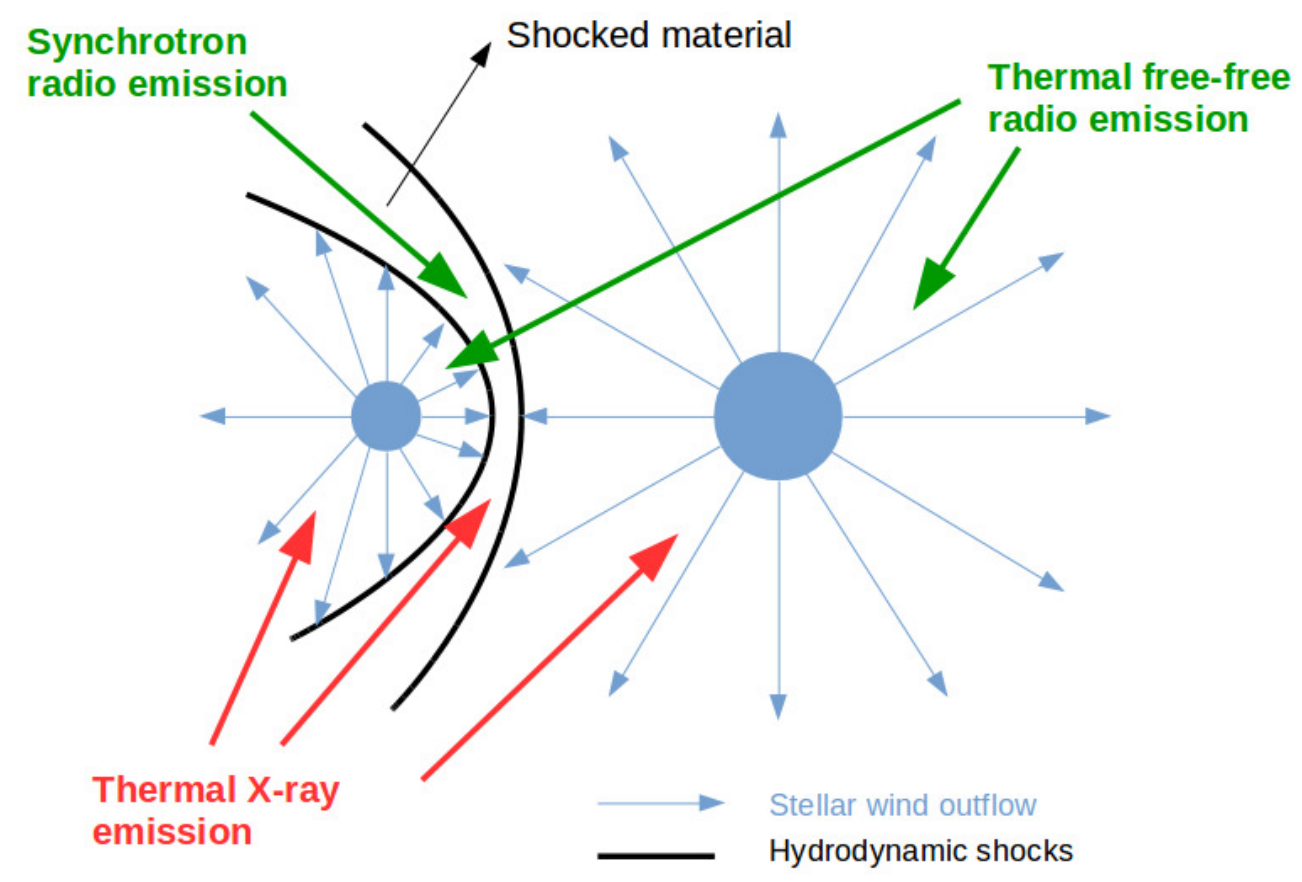

Figure 1: Schematic view of a colliding-wind binary.

In the context of wind collisions producing strong hydrodynamic shocks, particle acceleration is believed to be Diffusive Shock Acceleration (DSA). It consists of an iterative process allowing charged particles crossing the velocity jump of the shocks to achieve relative energy gains of the order of V/c (V being the amplitude of the velocity jump, and c the speed of light). At every iteration, the escape probability is not zero, resulting in a power law distribution of relativistic particles (Fermi 1949, Bell 1978, Drury 1983). In the specific context of DSA operating in CWBs, several relevant studies deserve to be mentioned, including Eichler \& Usov (1993), Pittard \& Dougherty (2006), Reimer et al. (2006) or Reitberger et al. (2014).

Synchrotron radio emission is most probably produced close to the wind-wind interaction region, where the relativistic electrons are accelerated. The flux density of this non-thermal emission scales as $\mathrm{S}_{\nu} \propto \nu^{-(p-1) / 2}$, with $\mathrm{p}$ being the index of the electron distribution. However, individual stellar winds are also radio emitters but this emission is of thermal origin. This thermal free-free emission is characterized by a flux density spectrum scaling as $\mathrm{S}_{\nu} \propto \nu^{0.6}$, assuming a spherical wind with the density scaling as $1 / \mathrm{r}^{2}$ (Wright \& Barlow 1975, Panagia \& Felli 1975). Consequently, the overall radio spectrum from PACWBs is composite, with thermal and non-thermal contributions (see Fig. 2). The different trends (given the different sign of the slopes) adopted by the thermal and non-thermal contributions as a function of frequency suggest that observations at lower frequencies are more adequate 
to identify synchrotron radiation. At higher frequencies, the spectrum may be dominated by thermal emission. However, even at higher frequencies the presence of a synchrotron contribution is expected to affect significantly the measured spectral index. It should be noted that the shocked plasma in the wind-wind interaction region could also provide an additional thermal contribution, resulting in a measured spectral index slightly lower than 0.6, even in the absence of any non-thermal contribution (Pittard et al.2006). As a conservative limit, it is thus considered that the radio spectrum includes a non-thermal contribution if the measured spectral index is $\leq 0.3$ (De Becker \& Raucq 2013).

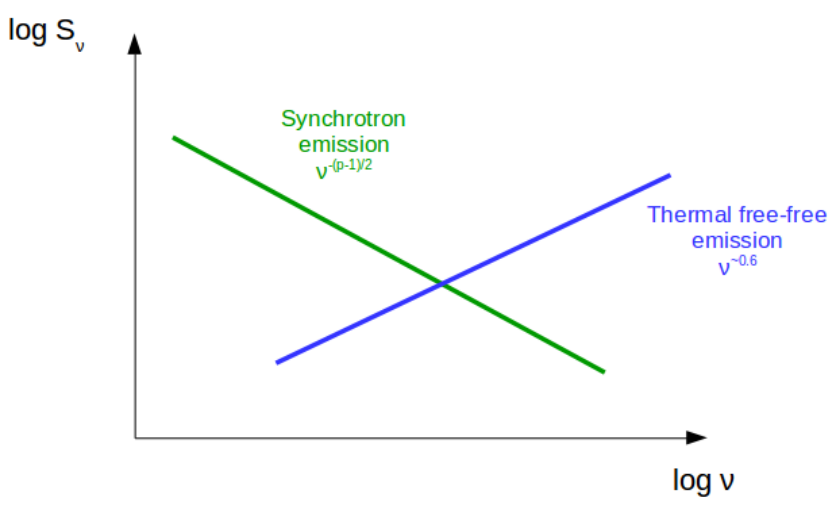

Figure 2: Schematic representation of the thermal and nonthermal components contributing to the radio spectrum of a PACWB.

Considering the binary nature of PACWBs, one may expect some variability in the measured radio spectrum as a function of the orbital phase. In this context, the variability time-scale is the orbital period. First of all, most systems are significantly eccentric, causing changes of the physical properties of the wind-wind interaction region where the synchrotron emission is produced. Second, the stellar wind material is optically thick for radio photons. Free-free absorption will therefore modulate the overall emission with an amplitude that will depend on the orientation of the system and on the orbital phase. A significant variability in the radio spectrum is also a signature for a non-thermal emission component produced in the colliding winds region. Finally, the synchrotron emission can be quite bright and it is produced on top of the thermal components from the stellar winds. Consequently, the typical brightness temperature of non-thermal sources will be significantly higher than that of purely thermal emitters. This constitutes another indication of the existence of a non-thermal contribution.

Massive binaries in general - and Particle-Accelerating Colliding-Wind Binaries in particular are responsible for a significant feedback on the interstellar medium. Not only because of their high UV/Visible luminosity and of their important release of mechanical energy through their strong stellar winds, but also by the way of their potential contribution to the production of galactic cosmic-rays. All these aspects of massive star physics have a significant impact on the physics of interstellar clouds (see e.g. De Becker 2014).

\section{The catalogue of Particle-Accelerating Colliding-Wind Bina- ries (PACWBs)}

Synchrotron emission is thus the main tracer of particle acceleration in CWBs. Most PACWBs in the catalogue (De Becker \& Raucq 2013) have indeed been identified through indications of nonthermal emission in the radio spectrum. So far, about 40 systems have been identified to be particle accelerators among CWBs. Stellar categories found in the catalogue include O-type stars, Wolf-Rayet stars, and a few 'transitional' objects (in evolutionary stages between $\mathrm{O}$ and WR types). An on-line version of the content of the catalogue can be consulted through the following link: 


\section{http://www.astro.ulg.ac.be/ debecker/pacwb}

Previous censuses used to separate systems at different evolutionary stages, with different lists for O-type and WR-type objects (e.g. Dougherty \& Williams 2000, De Becker 2007, Benaglia 2010). However, it turns out that for all these stellar categories, basically the physics is the same: one is dealing with objects with strong winds, with similar geometries, with wind collisions producing shocks where particles can be accelerated, with subsequent production of non-thermal radiation. The production of the catalogue was thus motivated by a strong need to unify these objects into a unique class, in order to achieve a more global and relevant view of their common physics.

Positions of the dominating star of each system (with the strongest stellar wind) in the diagram plotting the mass loss rate (in solar mass per year) and the terminal velocity (in $\mathrm{km} / \mathrm{s}$ ) in De Becker \& Raucq (2013) cover a wide parameter space, including the complete range of values typical of O-type and WR stars. However, the stellar parameters of the companions are still lacking in several systems, deserving future investigations to clarify this information. Concerning the orbital periods, the lower limit on the period seems to be located at 'a few weeks' with periods up to several decades. Here again, for many systems, the orbital period is not well-determined yet, and the lack of information is even more severe for orbital elements.

\section{The role of multiwavelength observations}

\subsection{Spectral classification and orbital parameters}

As we are dealing with binary or higher multiplicity systems, multiplicity investigations are strongly needed. Fundamentally, the determination of the orbital period is crucial as it provides the variability time-scale of the radio spectrum. The determination of the orbital parameters is also very important for the interpretation of the behavior PACWBs. If possible, the full characterization of the threedimension orbit constitutes a significant asset. Typically, one can expect free-free absorption to inhibit the capability to detect synchrotron radiation during some parts of the orbit because of orientation effects, and a 3-D view of the orbit is important to interpret the influence of this process.

In addition, the determination of the spectral classification of the stars is also important. Without any clear knowledge of the stellar parameters, it is quite difficult to estimate (i) the amplitude of the thermal emission components in the composite spectrum, and (ii) the impact of free-free absorption at a given orbital phase. One has also to remember that the source of energy feeding the non-thermal processes is basically the kinetic power of the stellar winds, determined on the basis of the mass loss rate and terminal velocities. No assessment on the energy budget of PACWBs could be made without prior knowledge of the kinetic power made available by the stars.

Spectroscopic investigations and high angular resolution imaging techniques applied to PACWBs allowed to determine orbital parameters and spectral classification of many systems. Relevant recent examples are the publications by Sana et al. (2011), De Becker et al. (2012), Sana et al. (2013), or Le Bouquin et al. (2017). In the context of the near future operation of the Devasthal Optical Telescope (DOT), spectroscopic observations of PACWBs could be made to determine/clarify spectral classifications (with a strong requirement for a medium/high resolving power), and feed spectroscopic time series aimed at following orbits of CWBs on time-scales of months and years to determine orbital elements. In particular, repeated spectroscopic observations of massive stars over various time-scales are needed to identify new binaries, potentially candidates to be observed at other wavebands to search for indication of particle acceleration (see Sections 3.2 and 3.3). For a first exploration of the multiplicity of massive stars, observations don't need to be time-constrained and would therefore be easy to schedule. 


\subsection{Identifying new members through radio observations}

If one wants to upgrade the catalogue, the identification of additional members is needed. To do so, radio observations constitute a privileged approach (see Sect. 1). The primary step consists of selecting likely candidates for future radio observations. Following a recent study addressing this specific issue, there is a priori no reason to focus on a restricted subset of CWBs (De Becker et al. 2017). In short, there is no need to (i) investigate strongly magnetic objects, (ii) focus on the systems with huge wind kinetic power (though, late-type main-sequence objects are less favorable), (iii) to investigate objects with very strong wind collisions, and (iv) to restrict investigations to a narrow range of orbital periods. At this stage, no specific parameter/ingredient seems to be required to discriminate between Particle-Accelerating and non-Particle-Accelerating CWBs. As a result, plenty of systems deserve to be considered.

Observation strategies should include:

- Repeated radio observations, to address the issue of the variability over time bases long enough to cover the orbital period. Adequate examples are WR140 (Dougherty et al. 2005), HD 168112 (Blomme et al. 2005), HD 167971 (Blomme et al.2007), or Cyg OB2 \#8A (Blomme et al. 2010).

- Measurements, at least, at two frequencies (to determine spectral indices). This is for instance the approach adopted by Benaglia et al. (2001) for a sample of Southern objects.

- Observations at longer wavelengths, where synchrotron emission should dominate the spectrum (e.g. 20 to $100 \mathrm{~cm}$ with the Giant Meterwave Radio Telescope in India),

- If feasible, consider Very Long Baseline Interferometry imaging to resolve spatially the nonthermal and the thermal emission components of the system. Recently, this technique was applied to the case of the very long period O-type binary HD 93129A (Benaglia et al. 2015).

A recent detailed discussion about observation strategies adequate for PACWB studies has been published by De Becker et al. (2017).

\subsection{Identifying new members through high energy observations}

The presence of high energy particles calls upon dedicated studies in the high energy domain. In the presence of relativistic electrons, the strong photospheric UV/visible radiation field can feed an inverse Compton (IC) scattering process. Basically, lower energy photons are up-scattered to high energies, taking away a fraction of the energy of the relativistic electrons they are interacting with. This process, of non-thermal nature, should produce a power-law spectrum, with typical photon energies directly related to the incident photon energy and to the square of the Lorentz factor of the relativistic electron (Blumenthal \& Gould 1970). Such a non-thermal high energy emission component could thus be considered as a complementary indicator to identify particle accelerators among CWBs.

An important point deserving to be mentioned is that CWBs are known to be thermal X-ray emitters (see Fig. 1). On the one hand, individual stellar winds are not steady. Instabilities in the linedriving mechanism cause the out-flow to be fragmented, with parcels of material moving at different speeds. Collisions between different components of the moving plasma will produce hydrodynamic shocks in the winds, with pre-shock velocities up to several $100 \mathrm{~km} / \mathrm{s}$. The post-shock temperature will reach values up to a few $10^{6} \mathrm{~K}$. Such a warm plasma will produce thermal X-rays (Lucy \& White 1980, Feldmeier et al. 1997). When stellar winds collide, the shocks (see Fig. 1) are characterized by pre-shock velocities of the order of the terminal velocity, provided the collision occurs at 
a distance large enough to allow the winds to reach their terminal velocities (for PACWBs identified so far this condition is rather well fulfilled). As a result, the post-shock temperature will reach values of few $10^{7} \mathrm{~K}$, with production of thermal X-ray photons as well (Stevens et al. 1992, Pittard \& Parkin 2010). Consequently, the soft X-ray spectrum (below $10 \mathrm{keV}$ ) is dominated by thermal emission. This strongly motivates to explore hard X-rays (i.e. above $10 \mathrm{keV}$ ) to search for an IC scattering component in the high energy spectrum.

One should keep in mind that some requirements must be satisfied for hard X-ray observations of CWBs:

- Good sensitivity. Non-thermal X-ray emission from CWBs is expected to be quite weak, with respect to that of other classes of objects such as X-ray binaries. For instance, the INTEGRAL satellite was used to search for hard X-ray emission from a sample of CWBs in the Cygnus region. This attempt resulted in no detection, and only upper limits on the putative inverse Compton scattering emission could be determined (De Becker et al.2007). Actually, INTEGRAL lacks the required sensitivity to detect most of these sources. The only exception is $\eta$ Car, an evolved binary that has been detected in hard X-rays first with BeppoSax (Viotti et al. 2004), and then with INTEGRAL (Leyder et al. 2008) and Suzaku (Sekiguchi et al. 2009).

- Good angular resolution. Fields where massive stars are located can be somewhat crowded, and it introduces severe issues of source confusion if the angular resolution is not good enough. This is especially critical in hard X-rays, where dealing with the issue of the background is of high importance. For instance, the putative detection of a non-thermal hard X-ray tail in the spectrum of the massive WR + O binary WR 140 is certainly attributable to a nearby background Seyfert galaxy (Sugawara et al. 2015).

- Spectral analysis capability. This is important to derive the spectral index of the high energy emission, directly related to the index of the relativistic electrons population.

Beside X-rays, it should also be mentioned that $\eta$ Car has firmly been identified as a $\gamma$-ray source, on the basis of AGILE (Tavani et al. 2009) and Fermi (Abdo et al. 2010, Farnier et al. 2011) observations. WR 11 might also be associated to another Fermi source (Pshirkov 2016). However, attempts to detect a sample of WR-type objects with Fermi led to non-detections (Werner et al. 2013). In the $\gamma$-ray domain, beside a potential high energy IC scattering tail likely to contribute, a hadronic process such as neutral pion decay following interactions of relativistic protons with thermal particles could be an important process. In particular, it is thought to be responsible for the $\gamma$-ray emission associated to $\eta$ Car (Farnier et al. (2011). Here again, it is referred to De Becker et al. (2017) for a detailed discussion of observation strategies and expectations from high energy observations of PACWBs.

\subsection{Towards understanding the non-thermal physics}

The modelling of the physics of PACWBs is a crucial aspect of this science topic. A substantial modelling effort has been developed during the last past years. Among others, let's mention the recent work by del Palacio et al. (2016), dedicated to the very long period binary HD 93129A. This study allowed to made some predictions for observations, and potential detectability of the system with the NuStar satellite (Hailey et al.2010), in hard X-rays.

In addition, the modelling allows to produce theoretical Spectral Energy Distributions aimed at being confronted to actual measurements across the electromagnetic spectrum. It is notably interesting to note that observations in the $\mathrm{MeV}$ range are especially interesting to lift degeneracies between 
models. The present lack of $\mathrm{MeV}$ observatory with the required sensitivity is an issue, that could be solved in the near future with a project such as AstroMeV'

\section{Concluding remarks}

Particle acceleration in colliding-wind binaries should not be a scarce phenomenon. The study of Particle-Accelerating Colliding-Wind Binaries is now switching to a new regime: from a few individual studies of massive binaries, one can now consider to study a real class of objects covering a rather wide parameter space (both from the stellar wind and the orbital points of view).

The role of multi-wavelength observations is highly important:

I. Optical (and IR) observations : useful for the determination of the orbital parameters and for the characterization of the nature of the components in the binary - multiple - systems. This information is the starting point to define observations strategies in other wavebands.

II. Radio observations : crucial to identify synchrotron radio emitters, as is it the main tracers of particle acceleration in massive binaries.

III. High energy (X-ray and gamma-ray) investigations: this constitutes a complementary (and independent) approach to search for non-thermal emission processes indicating a particle acceleration process is at work.

Adequate observational efforts (and strategies) are strongly needed to address the question of the fraction of PACWBs among CWBs. To date, the census of PACWBs is strongly influenced by severe observational biases. On the basis of simple arguments, one can say there is a priori no reason to consider that particle acceleration in massive binaries couldn't be much more frequent than actually suggested by the size of the present catalogue. This motivates to develop further the search for the identification of additional PACWBs.

Aside from the importance of particle acceleration and non-thermal processes in general for the understanding of the physics of colliding-wind binaries, one should emphasize the potential role played by these objects in the production of galactic cosmic-rays, at least for the lower energy part of the cosmic-ray spectrum. Though the main contributors should be supernova remnants, one should keep in mind that supernova remnants are efficient at accelerating particle for time-scales of the order of $10^{4} \mathrm{yr}$. Alternatively, PACWBs should be able to do so as long as both components do not go through the supernova episode. For massive stars, the typical time-scale is of the order of a few $10^{6} \mathrm{yr}$, up to about $10^{7} \mathrm{yr}$. Put together, the contributions from all PACWBs in the Milky Way could provide a not so negligible contribution to the low energy galactic cosmic-ray spectrum, provided the fraction of PACWBs among CWB is not too weak. In this context, the feedback of PACWBs on the whole galactic environment may be significant, motivating further to investigate the issue of the determination of this fraction.

\section{Acknowledgements}

This research is supported in part by a ULiège 'Crédit classique: DARA'. The ADS has been used for the bibliography.

\footnotetext{
${ }^{1}$ http://astromev.in2p3.fr/?q=aboutus/pact
} 


\section{References}

Abdo A. A., Ackermann M., Ajello M. et al. 2010, ApJS, 187, 460

Bell A. R. 1978, MNRAS, 182, 147

Benaglia P. 2010, in High Energy Phenomena in Massive Stars, eds. J. Mart, P. L. Luque-Escamilla, \& J. A. Combi, ASP Conf. Ser., 422, 111

Benaglia P., Cappa, C. E., Koribalski, B. S. 2001, A\&A, 372, 952

Benaglia P., Marcote B., Moldón J. et al. 2015, A\&A, 579, A99

Blomme R., Van Loo S., De Becker M. et al. 2005, A\&A, 436, 1033

Blomme R., De Becker M., Runacres M. C., van Loo S., Setia Gunawan, D. Y. A. 2007, A\&A, 464, 701

Blomme R., De Becker M., Volpi D., Rauw, G. 2010, A\&A, 519, A111

Blumenthal P., Gould G. E. 1970, Rev. Mod. Phys., 42, 237

Crowther P. A. 2007, ARAA, 45, 177

De Becker M. 2007, A\&ARv, 14, 171

De Becker M. 2014, Ap\&SS, 350, 237

De Becker M., Raucq F. 2013, A\&A, 558, A28

De Becker M., Rauw G., Pittard J. M. et al. 2007, A\&A, 472, 905

De Becker M., Sana H., Absil O., Le Bouquin J.-B., Blomme R. 2012, MNRAS, 423, 2711

De Becker M., Benaglia P., Romero G. E., Peri C. S. 2017, A\&A, 600, A47

del Palacio S., Bosch-Ramon V., Romero G. E., Benaglia P. 2016, A\&A, 591, A139

Dougherty S. M., Williams P. M. 2000, MNRAS, 319, 1005

Dougherty S. M., Beasley A. J., Claussen M. J., Zauderer B. A., Bolingbroke N. J. 2005, ApJ, 623, 447

Drury L. O'C. 1983, Rep. Prog. Phys., 46, 973

Eichler D., Usov V. 1993, ApJ, 402, 271

Farnier C., Walter R., Leyder J.-C. 2011, A\&A, 526, A57

Feldmeier A., Puls J., Pauldrach A. W. A., 1997, A\&A, 322, 878

Fermi E., 1949, Physical Review, 75, 1169

Hailey C. J., An H., Blaedel K. L. et al. 2010, Space Telescopes and Instrumentation 2010: Ultraviolet to Gamma Ray. Edited by Arnaud M., Murray S. S., Takahashi T., Proceedings of the SPIE, Volume 7732, article id. $77320 \mathrm{~T}$

Le Bouquin J.-B., Sana E., Gosset E. et al. 2017, A\&A, 601, A34

Leyder J.-C., Walter R., Rauw G. 2008, A\&A, 477, L29

Lucy L. B., White R. L. 1980, ApJ, 241, 300

Muijres L. E., Vink J. S., de Koter A., Müller P. E., Langer N. 2012, A\&A, 537, A37

Panagia N.,, Felli M. 1975, A\&A, 39, 1

Pittard J. M., Dougherty S. M. 2006, MNRAS, 372, 801

Pittard J. M., Parkin E. P. 2010, MNRAS, 403, 1657

Pittard J. M., Dougherty S. M., Coker R. F., OConnor E, Bolingbroke N. J. 2006, A\&A, 446, 1001

Pshirkov M. S. 2016, MNRAS, 457, L99

Reimer A., Pohl M., Reimer O. 2006, ApJ, 644, 1118

Reitberger K., Kissmann R., Reimer A., Reimer O. 2014, ApJ, 789, 87

Sana H., Evans C. J. 2011, in IAU Symp. 272, eds. C. Neiner, G. Wade, G. Meynet, \& G. Peters, 474

Sana H., Le Bouquin J.-B., De Becker M., et al. 2011, ApJ, 740, L43

Sana H., de Mink S. E., de Koter A. et al. 2012, Science, 337, 444

Sana H., Le Bouquin J.-B., Mahy L. et al. 2013, A\&A, 553, A131

Sekiguchi A., Tsujimoto M. Kitamoto S. et al. 2009, PASJ, 61, 629

Stevens I. R., Blondin J. M., Pollock A. M. T. 1992, ApJ, 386, 265

Sugawara Y., Maeda Y., Tsuboi Y. et al. 2015, PASJ, 67, 121

Tavani M., Sabatini S., Pian E. et al. 2009, ApJ, 698, L142

Viotti R. F., Antonelli L. A., Rossi C., Rebecchi S. 2004, A\&A, 420, 527

Werner M., Reimer O., Reimer A., Egberts K. 2013, A\&A, 555, A102

Wright A. E., Barlow M. J. 1975, MNRAS, 170, 41 